\title{
Mapping Polarity, Toroidal Order, and the Local Energy Landscape by 4D-STEM
}

Kayla X Nguyen ${ }^{1}$, Yi Jiang ${ }^{2}$, Michael C. Cao ${ }^{1}$, Prafull Purohit ${ }^{6}$, Ajay K. Yadav ${ }^{3}$, Javier Junquera ${ }^{4}$, Mark W. Tate ${ }^{6}$, Sol M. Gruner ${ }^{2,5,6,7}$, Ramamoorthy Ramesh ${ }^{3}$, Sayeef Salahuddin ${ }^{8}$ and David A. Muller ${ }^{1,5}$

${ }^{1}$ School of Applied and Engineering Physics, Cornell University, Ithaca, NY, USA

2.Department of Physics, Cornell University, Ithaca, NY, USA

3.Department of Material Science and Engineering, University of California, Berkeley, CA, USA

4.Department of Physics, University of Cantaberia, Spain

${ }^{5}$ Kavli Institute at Cornell for Nanoscale Science, Ithaca, NY USA

${ }^{6}$ Laboratory of Atomic and Solid State Physics, Cornell University, Ithaca, NY, USA

7. Cornell High Energy Synchrotron Source (CHESS), Cornell University, Ithaca, NY, USA

${ }^{8}$ Department of Electrical Engineering and Computer Sciences, University of California, Berkeley, CA, USA

Ferroelectric polarization vortex arrays in $\mathrm{PbTiO}_{3} / \mathrm{SrTiO}_{3}$ superlattice [1] show topological features with toroidal order, providing a ferroelectric analog to magnetic textures like Skyrmions. Polar displacements are usually measured with aberration-corrected annular dark field scanning transmission electron microscopy (ADF-STEM), which limits the field of view and sensitivity where picometer sensitivity in real space makes it very susceptible to scan noise and distortions. Here, we describe a 4-dimensional diffraction imaging methodology to quantitatively image the physical properties of the polarization vortices - polarity, toroidal order and the local energy landscape - giving us multiple natural order parameters of the system. All of this is enabled by the high-speed ( $0.86 \mathrm{~ms}$ readout), high-dynamicrange $\left(10^{6}: 1\right)$ electron microscope pixel array detector (EMPAD) [2] developed at Cornell which captures the full convergent beam electron diffraction (CBED) pattern at each scan position (Figure 1a). From which, we can uncover components of the macroscopic polarization.

We show that the probability current flow, $\langle\boldsymbol{P}\rangle$, of conjugate diffraction disks tracks the changes in polarity and when reconstructed into an image, provides a direct visualization of the polarization vortices (Figure 1b-c). The electric toroidal moment of the sample supplies a torque to the electron beam resulting in a transfer of orbital angular momentum (OAM) to the electron beam that is recorded on the EMPAD (Figure 1d-e, OAM in units of $\hbar$ ). By measuring polarity, $p$, and electric field, $E$, independently and simultaneously on the EMPAD, we use the relation $U=\vec{p} \cdot \vec{E}$, to extract the local electrostatic energy landscape of our system. The second derivative of the local energy with respect to polarity gives the capacitance of that region, showing regions of negative differential capacitance in the core of the vortices. These are the key physical parameters needed to design scalable ferroelectric devices, and adds functionality to the electron microscope at a spatial resolution inaccessible by any other tool [3].

\section{References:}

[1] Yadav, A. K. et al, Nature, 530 (2016), p. 198-201.

[2] Tate W. M. et al, Microscopy and Microanalysis. 22 (2016), p. 237-249.

[3] Supported by NSF MRSEC program (DMR 1719875), Kavli Institute at Cornell. 


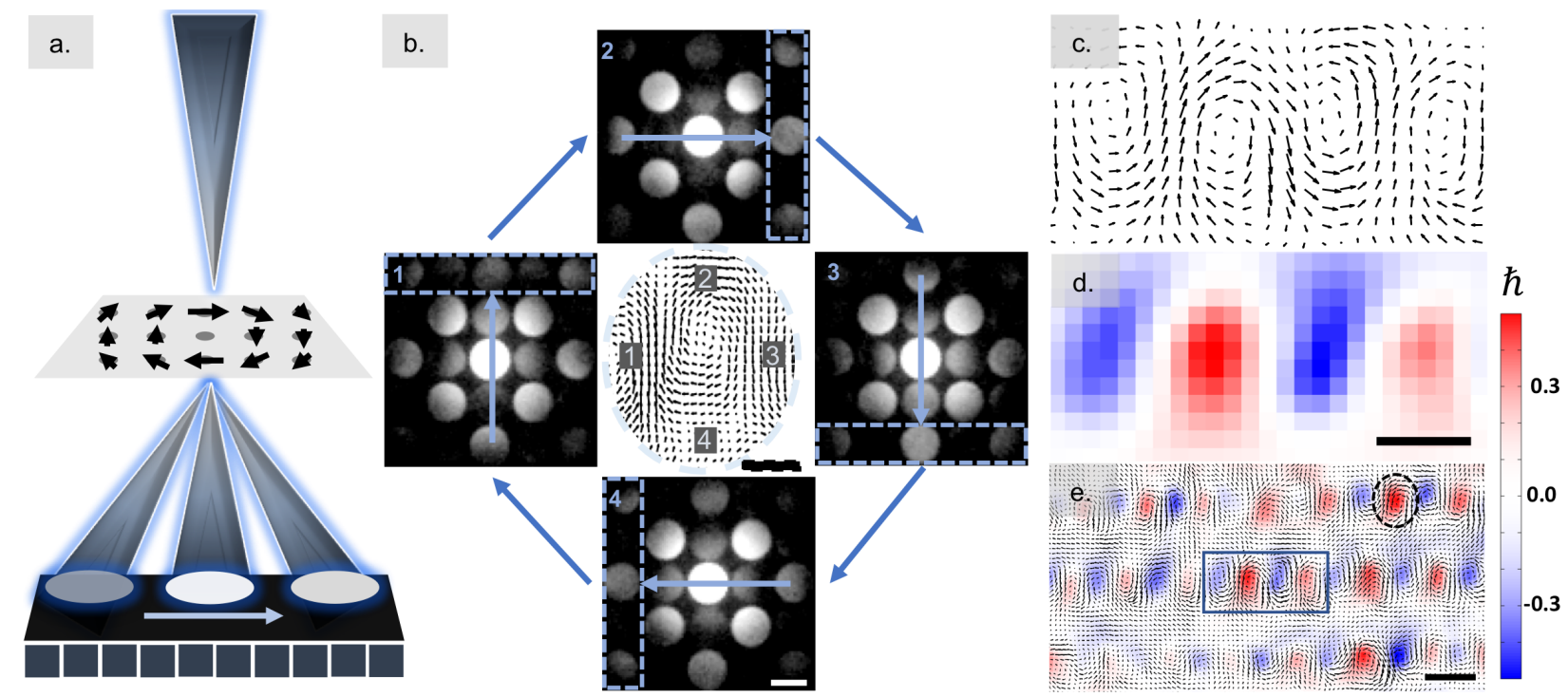

Figure 1. (a) Schematic of electron microscope pixel array detector (EMPAD) placed in the diffraction plane to record the full angular scattering distribution from an electron beam focused onto a sample. Here, the vortex is circled and different regions of the vortex (labeled 1-4) are shown with their respective convergent beam electron diffraction (CBED) patterns (1-4). We observed that the changes in intensity of the CBED patterns correlate with its location on the vortex. We obtain the change in orbital angular momentum (OAM) of the electron beam in units of Planck's constant, $\hbar$ and compared our polarity map in (c) to our toroidal moment in (d). Larger field of view is shown in (e). Scale bar in (b) is $6 \mathrm{mrad}$ for the CBED patterns and $2 \mathrm{~nm}$ for the polarization vortex. Black scale bar in (d) is $2 \mathrm{~nm}$ and (e) is $5 \mathrm{~nm}$. Black dashed circle in (e) shows the polarization vortex seen in (b), and the boxed region in (e) represent the polarization vortices in (c) and (d).

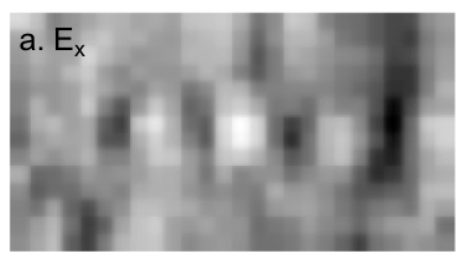

b. $E_{z}$
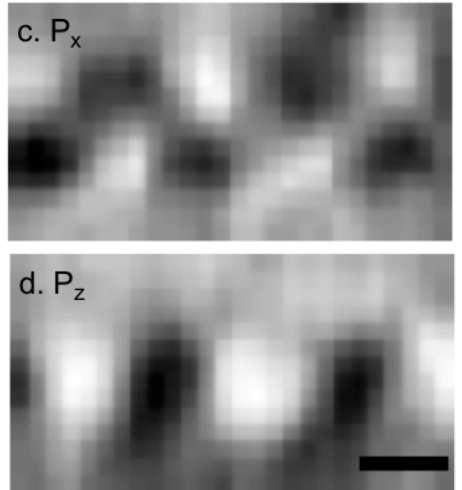

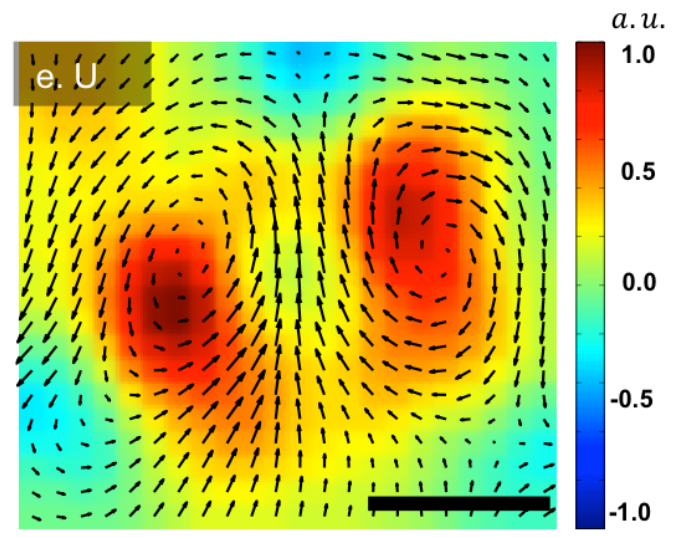

a.u.

0.5

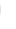
5 .

Figure 2. Reconstruction of the (a) electric field in $x,\left(E_{x}\right)$ and (b) $z,\left(E_{z}\right)$, using the (00) disk which is susceptible to all changes except polarization. Measurement of the polarity using the probability current flow in $\{200\}$ diffraction disks to reconstruct polarity changes in $(c) x,\left(P_{x}\right)$, and the [020] diffraction disk to reconstruct polarity changes in $(d) z,\left(P_{z}\right)$. By calculating the electric field and polarity, we can then derive the internal energy $U$ of the structure seen in (e). Black scale bar in (d) is $2 \mathrm{~nm}$ and (e) is 4 nm. 